\title{
Pneumoperitoneum following endoscopic mucosal resection without perforation of the colon
}
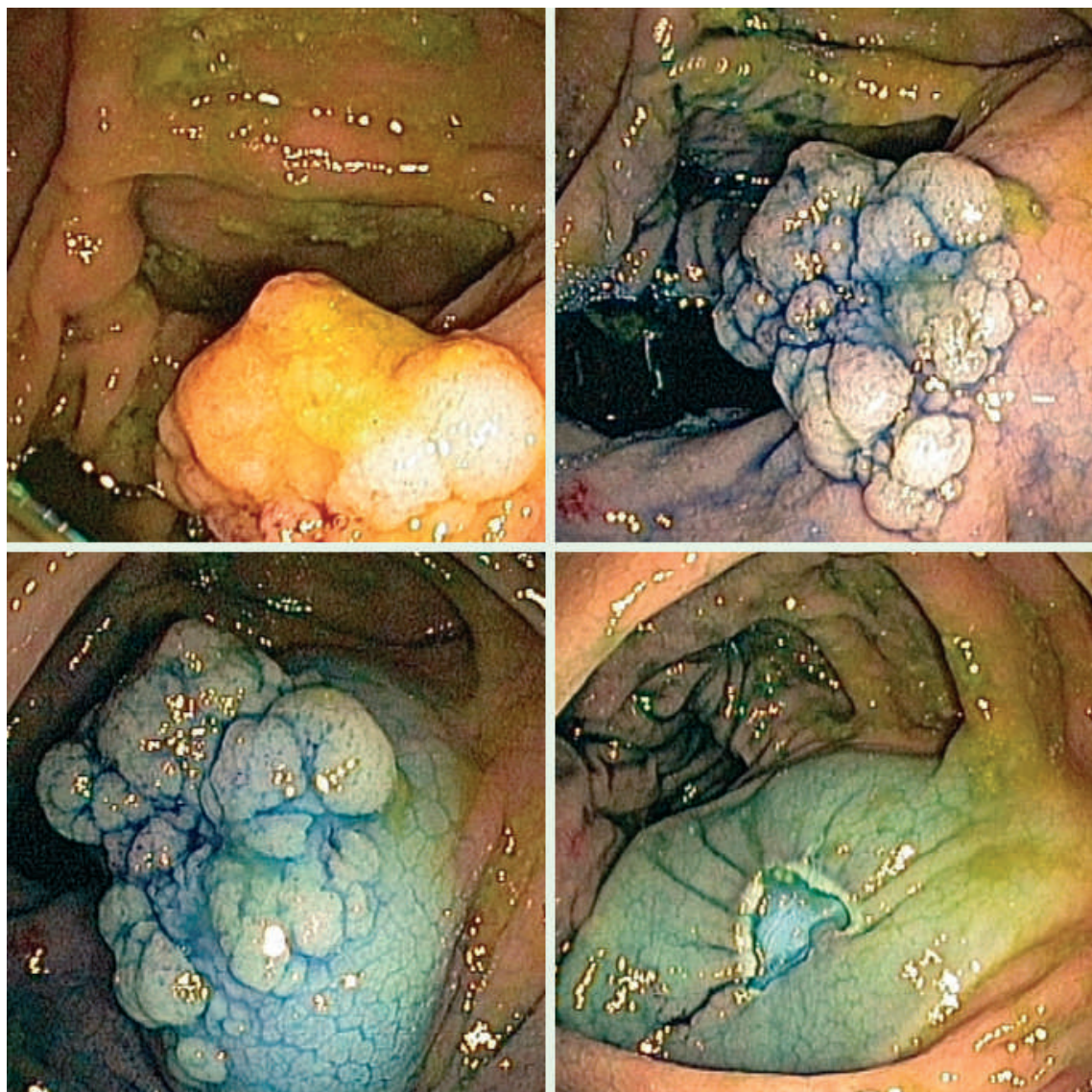

Fig. 1 Adenoma of the cecum and endoscopic mucosal resection after indigo carmine staining and lifting by submucosal injection.

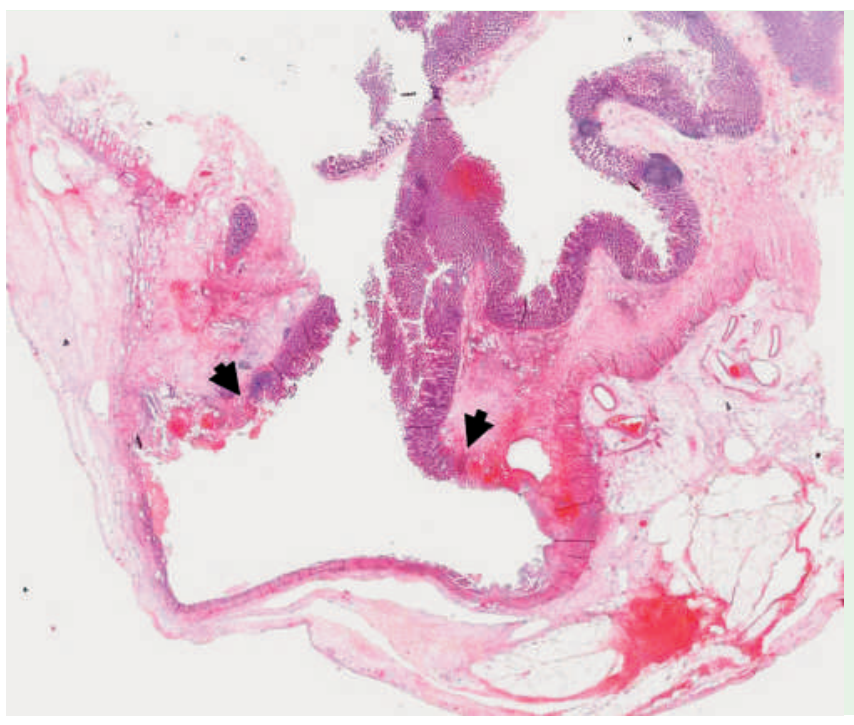

Fig. 3 Whole mount view on the histological section of the endoscopic mucosal resection site. A large defect in the inner layers of the colonic wall extending to the subserosa is seen, but still a small rim of intact subserosa and serosa is found. Arrows indicate the margins of the site of resection. Hematoxylin and eosin stain.

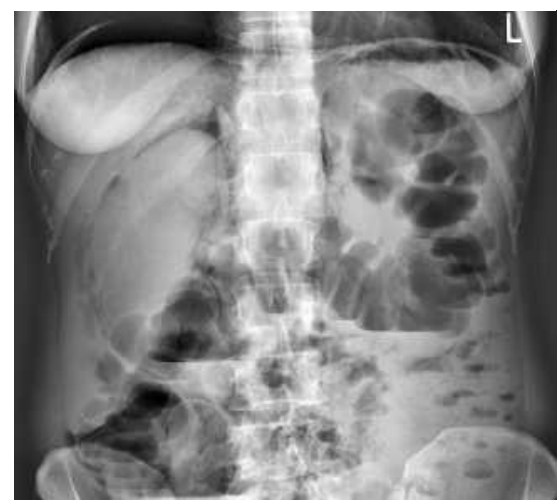

Fig. 2 Abdominal radiograph showing a pneumoperitoneum on the day following endoscopic mucosal resection.

In an otherwise healthy 39-year-old female patient with a family history of colon carcinoma, a sessile adenoma with low-grade intraepithelial neoplasia of approximately $2.5 \mathrm{~cm}$ was found in the cecum during screening colonoscopy. After indigo carmine staining the adenoma was lifted by submucosal injection of $8 \mathrm{~mL}$ normal saline containing epinephrine $1: 100000$, and endoscopic mucosal resection (EMR) was performed using a standard polypectomy snare ( $\bullet$ Fig. $\mathbf{1}$ ). The day after the procedure the patient developed generalized abdominal defense, and an abdominal radiography was performed showing a pneumoperitoneum ( $\bullet$ Fig. 2). Because of a suspected perforation of the cecum the patient underwent explorative laparotomy and ileocecal resection the same day. Macroscopically, only a hematoma of the cecal wall was seen, and neither perforation of the cecum nor local peritonitis was observed intraoperatively, and thus primary anastomosis was performed. The patient was discharged in good health 5 days later. Histopathological analysis of the resectate revealed a defect at the site of mucosal resection down to the muscularis propria, with granulocytic infiltrates and a pneumatosis of the cecal wall ( $\bullet$ Fig. 3 and $\odot$ Fig. 4). 


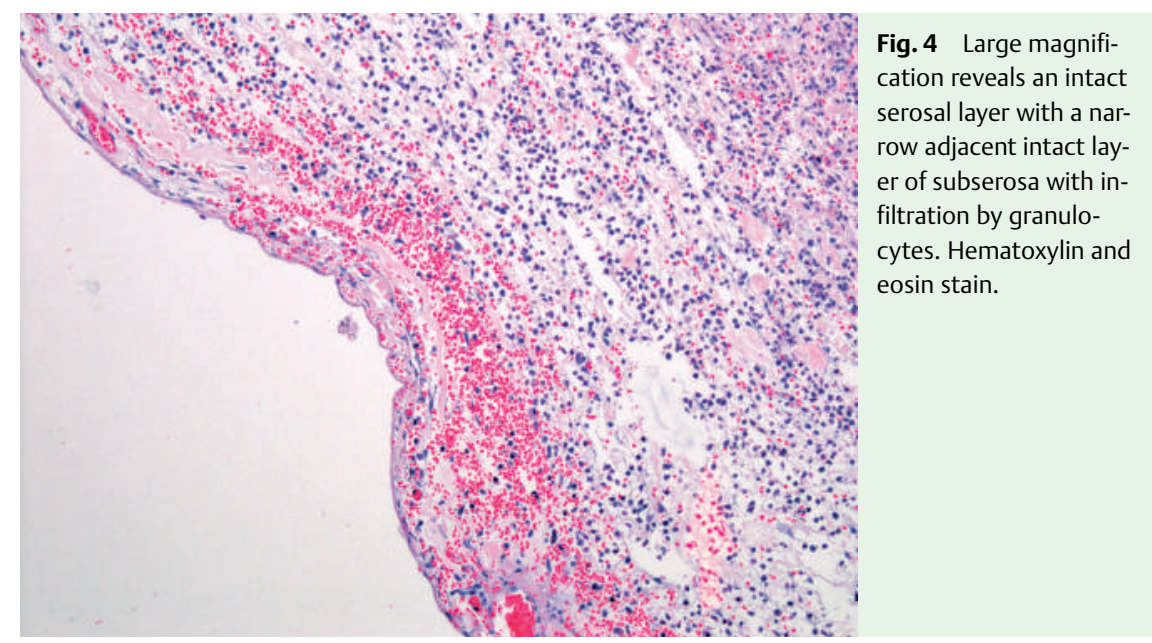

Perforation of the colon is a common complication after EMR, with an incidence of up to $0.5 \%$ [1] and frequently requiring surgical repair [2]. We report here a case where clinical and radiological signs of perforation following polypectomy were present, although no transmural opening of the cecal wall was observed at laparotomy or histopathologically. We conclude that the presence of a radiologically proven pneumoperitoneum does not necessarily indicate perforation of the colon, but might merely reflect translocalization of inflated air through the thinned colonic wall. Caution should be given to the evaluation of the clinical relevance of these symptoms and the indi-

\section{Eisenbach ${ }^{1}$, H. Bläker ${ }^{2}$, W. Stremmel ${ }^{1}$, P. Sauer ${ }^{1}$, A. Schaible ${ }^{3}$}

1 Department of Gastroenterology, University of Heidelberg, Heidelberg, Germany

2 Department of Pathology, University of Heidelberg, Heidelberg, Germany

3 Department of General Surgery, University of Heidelberg, Heidelberg, Germany

\section{References}

1 Frühmorgen P, Pfähler A. Komplikationen bei 39397 endoskopischen Untersuchungen - eine 7-jährige prospektive Dokumentation über Art und Häufigkeit. Leber Magen Darm 1990; 1: 20 - 32

2 Cobb WS, Heniford BT, Sigmon LB et al. Colonoscopic perforations: incidence, management, and outcomes. Am Surg 2004; 70: $750-758$

3 Iqbal CW, Chun YS, Farley DR. Colonoscopic perforations: a retrospective review. J Gastrointest Surg 2005; 9: 1229-1236

Bibliography

DOI $10.1055 / \mathrm{s}-2007-995493$

Endoscopy 2008; 40: E64 -E65

(c) Georg Thieme Verlag KG Stuttgart · New York . ISSN 0013-726X

\section{Corresponding author}

C. Eisenbach, MD

Department of Gastroenterology

University of Heidelberg

Im Neuenheimer Feld 410

D-69120 Heidelberg

Germany

Fax: + 49-6221-566148

Christoph_Eisenbach@med.uni-heidelberg.de 